\title{
No news for Kerr-Schild fields
}

\author{
Boyko V. Ivanov \\ Institute for Nuclear Research and Nuclear Energy, \\ Tzarigradsko Shausse 72, Sofia 1784, Bulgaria
}

\begin{abstract}
Algebraically special fields with no gravitational radiation are described. Kerr-Schild fields, which include as a concrete case the Kinnersley photon rocket, form an important subclass of them.
\end{abstract}

PACS numbers: 04.20.J

\section{INTRODUCTION}

In 1951 Vaidya [1] generalized the Schwarzschild vacuum solution to a spherically symmetric "shining star" solution, emitting pure radiation. In 1969 Kinnersley [2] further generalized this solution to a photon rocket - a particle of arbitrary acceleration emitting an anisotropic jet of radiation. He noted that it belonged to the Kerr-Schild (KS) class of metrics, studied till then in vacuum [3] and in the presence of electromagnetic fields [4]. Later it was shown that the photon rocket is also a type D pure radiation solution from the Robinson-Trautman (RT) class (algebraically special expanding solutions without a twist (rotation)) [5]. In 1994 Bonnor [6] found that axially symmetric photon rockets do not emit gravitational radiation, which is strange for an accelerating particle. A suggestion was made, based on the linearised theory, that axial symmetry was responsible for this phenomenon [7]. However, an axially symmetric RT solution of type II was studied as a anisotropic perturbation of the photon rocket, still with no sign of gravitational radiation [8]. Another explanation was given [9], based on the well-known vanishing of the Einstein pseudo-energy-momentum tensor for KS fields [10]. It was extended to the Einstein-Maxwell field of a charged particle, undergoing arbitrarily accelerated motion [11].

The fact that the photon rocket is a member of the RT class was emphasized in [12, 13]. In the latter reference the Bondi-Sachs [14, 15] formalism was used and applied to axially symmetric RT pure radiation solutions. It was shown that the Kinnersley solution is the only one which does not radiate gravitationally. This put the study of photon rockets on firm basis, since different pseudo-tensors for KS metrics lead to different results [16]. Later the news function was calculated for any RT metric and it was found once more that the Kinnersley solution is the only one to emit just photon radiation 17, 18]. Rotating pure radiation KS fields (radiating Kerr metric) were given in [19, 20] and it was demonstrated [20] that only pure radiation contributes to the mass loss.

Finally, the Bondi mass and the news function were recently found for twisting algebraically special metrics in a paper 21], based on previous research of Tafel and co-authors 22, 23], where the RT class was discussed as a special case.

Meanwhile, all pure radiation KS fields with axial symmetry have been found explicitly [24]. Stephani [5, 25] proposed a more compact description of KS fields and rederived the results of Herlt, giving also an example of a non-axially symmetric solution. This approach has been developed further by the present author [26, 27, 28, 29].

In this paper we use the same approach to study fields with vanishing news function and consequently, with no gravitational radiation. The KS metrics form a large class of such solutions but do not exhaust all of them. The intersection between KS and RT fields is represented by the Kinnersley photon rocket, which explains why more general RT solutions always radiate gravitationally.

In Section II the notation for twisting algebraically special metrics is fixed and the expressions for the Bondi mass and the news function are given according to 21]. In Section III the condition for no news is discussed and different classes of metrics that satisfy it are given.

\section{THE BONDI MASS AND NEWS FUNCTION}

Pure radiation (aligned case) and vacuum algebraically special fields satisfy the Einstein equations

$$
R_{\mu \nu}=\Phi k_{\mu} k_{\nu}
$$

*Electronic address: boyko@inrne.bas.bg 
where $k^{\mu}$ is the multiple null eigenvector of the Weyl tensor and $\Phi \geq 0$, vanishing for vacuum solutions. In some coordinates $u . r, \xi, \bar{\xi}$ the interval reads $[\underline{5}]$

$$
d s^{2}=2 \omega(d r+W d \xi+\bar{W} d \bar{\xi}+H \omega)-2 \frac{r^{2}+\Sigma^{2}}{P^{2}} d \xi d \bar{\xi}
$$

where

$$
\begin{gathered}
\omega=d u+L d \xi+\bar{L} d \bar{\xi}, \quad \partial=\partial_{\xi}-L \partial_{u} \\
W=-(r+i \Sigma) L_{, u}+i \partial \Sigma, \quad \Sigma=\frac{i}{2} P^{2}(\partial \bar{L}-\bar{\partial} L) \\
H=-r(\ln P)_{, u}-\frac{m r+M \Sigma}{r^{2}+\Sigma^{2}}+P^{2} \operatorname{Re}\left[\partial\left(\bar{\partial} \ln P-\bar{L}_{, u}\right)\right]
\end{gathered}
$$

The functions $P, m, M$ are real and $L$ is a complex function of $u, \xi, \bar{\xi}$. The Einstein equations read [5], Eqs.(30.40)$(30.42)$

$$
\begin{gathered}
\partial(m+i M)=3(m+i M) L_{, u} \\
M=P^{3} I m \partial \partial \bar{\partial} \bar{\partial} V \\
P^{3}\left[P^{-3}(m+i M)\right]_{, u}=P^{4}\left(\partial+2 \partial \ln P-2 L_{, u}\right) \partial I-\frac{\eta^{2}}{2}
\end{gathered}
$$

where $P \equiv V_{, u}$ and

$$
\begin{gathered}
\Phi=\eta^{2} \rho \bar{\rho}, \quad \rho^{-1}=-(r+i \Sigma) \\
I=P^{-1}(\bar{\partial} \bar{\partial} V)_{, u}=\bar{\partial}\left(\bar{\partial} \ln P-\bar{L}_{, u}\right)+\left(\bar{\partial} \ln P-\bar{L}_{, u}\right)^{2}
\end{gathered}
$$

Eq. (8) is, in fact, a definition of $\eta^{2}$, the actual field equations being $(6,7)$, which are the same as in the vacuum case. Eqs. $(7,8)$ give also

$$
P^{3}\left(P^{-3} m-R e \partial \partial \bar{\partial} \bar{\partial} V\right)_{, u}=-P^{2}(\partial \partial V)_{, u}(\bar{\partial} \bar{\partial} V)_{, u}-\frac{\eta^{2}}{2}
$$

where $M$ is excluded and this condition may replace Eq. (8).

In 21] it was shown that $\bar{I}$ is the Bondi news function, which enters the tensor $n_{a b}$ and its derivative

$$
\begin{gathered}
n_{a b} d x^{a} d x^{b}=-2 P_{s}^{-1}\left(\partial \partial V d \xi^{2}+\bar{\partial} \bar{\partial} V d \bar{\xi}^{2}\right) \\
\left(n_{a b}\right)_{, u} d x^{a} d x^{b}=-2 \bar{I} d \xi^{2}-2 I d \bar{\xi}^{2}
\end{gathered}
$$

while the Bondi mass is given by

$$
M_{B}=m \hat{P}^{-3}-\frac{1}{2} \hat{P}^{-1}\left(\sigma^{0} \bar{\sigma}^{0}\right)_{, u}+P_{s}^{4} \operatorname{Re}\left[\hat{P}^{-1} I_{, u}(\partial \hat{u})^{2}-2 \partial I \partial \hat{u}\right]
$$

where

$$
\begin{array}{cc}
\hat{P} \equiv P P_{s}^{-1}, & P_{s}=1+\frac{1}{2} \xi \bar{\xi} \\
\sigma^{0}=P_{s} \partial \partial V, & \left(\sigma^{0}\right)_{, u}=P_{s} P \bar{I}
\end{array}
$$

and $\hat{u}$ is an approximate Bondi coordinate. Then Eq. (11) becomes the energy loss formula in the Bondi-Sachs approach. 


\section{FIELDS WITH NO NEWS}

The condition for vanishing news is obviously $I=0$. Eq. (10) gives in this case

$$
\partial \partial V=F(\xi, \xi)
$$

where $F$ is an arbitrary complex function. All, but the first term in the expression (14) for the Bondi mass, vanish

$$
M_{B}=\frac{m P_{s}^{3}}{P^{3}}
$$

Eq. (8) simplifies and its real part gives for the mass loss

$$
\left(M_{B}\right)_{, u}=-\frac{\eta^{2} P_{s}^{3}}{2 P^{3}}=-\frac{\eta^{2} M_{B}}{2 m}
$$

There is no gravitational radiation, the Bondi mass decreases due to the emission of photons and when $\eta=0$, it stays constant.

A large class of fields, satisfying the no news condition (17) are the Kerr-Schild fields, which can be defined by [25]

$$
\partial \partial V=0
$$

In this case the tensor $n_{a b}$ vanishes identically. One can choose a gauge with $m=1, M=0, L=i h_{\xi}$, where $h$ is real. Eq. (19) becomes

$$
\left(\ln V_{, u}\right)_{, u}=\frac{\eta^{2}}{6}
$$

which is, in fact, Eq. (32.79) from [5]. One can integrate it twice to find $V$ as a function of $\eta$. In the vacuum case $V$ must be linear in $u$ and the reality condition then uniquely fixes $h$. These solutions have been given already in 3 ] and include the Kerr black hole. In the pure radiation case the general solution is not known. Herlt [5, 25] has found the general axisymmetric solution. This includes the radiating Kerr metric [20]. An example of a non-axisymmetric solution was given in 25]. In all these metrics $\eta=\eta(u)$, so presumably it is more natural to base the classification of KS fields on the form of $\eta$ and not on the symmetries of the metric.

For RT solutions $L=0, M=0$ and the condition $I=0$ becomes

$$
P_{\xi \xi}=0, \quad P_{\bar{\xi} \bar{\xi}}=0
$$

Together they give the non-axisymmetric solution

$$
P=\alpha(u) \xi \bar{\xi}+\beta(u) \xi+\bar{\beta}(u) \bar{\xi}+\delta(u)
$$

where $\alpha, \delta$ are real. In general, $\alpha, \beta, \delta$ may be interpreted in terms of the acceleration of a particle moving along a spacelike, timelike or null world line [5], Sec. 28.3. When $K=2(\alpha \delta-\beta \bar{\beta})$ is positive we get the Kinnersley photon rocket. The case with no $u$-dependence represents the Vaidya shining star [1]. Consequently, the only RT solution with no gravitational radiation is the photon rocket, which belongs also to the KS class. This confirms the previous results [6, 9, 13, 17, 18].

Some general classes of twisting vacuum solutions have been found [5, 30] under the assumptions that $L, u-\partial \ln P$ and $m+i M$ are independent of $u$. Among them is the case with no news $I=0$ [5], Sec.29.2.4, which is solved explicitly. Thirty years ago it has been shown 31] that these are the only solutions which are non-radiative in the sense that the Weyl tensor for large $r$ behaves like $1 / r^{3}$. If the further condition $L_{, u}=0$ is imposed, the metric becomes independent of $u[\underline{5}]$, Sec.29.2.5. Then $P$ is given by Eq. (23) with constant $\alpha, \beta, \delta$ and the expressions for $m, M, L$ are given by Eq. (29.61) from [5]. The subclass $m+i M=$ const contains well-known type D solutions, such as Kerr and NUT, Kerr and Debney / Demianski four-parameter solution [32, 33]. When, in addition, $M=0$ we come again to the $\mathrm{KS}$ vacuum solutions [5].

[1] Vaidya P 1951 Proc. Indian Acad. Sci. A 33, 264

[2] Kinnersley W 1969 Phys. Rev. 186, 1335 
[3] Kerr R P and Schild A 1965 Proc. Symp. Appl. Math. 17, 199

[4] Debney G C, Kerr R P and Schild A 1969 J. Math. Phys. 10, 1842

[5] Stephani H, Kramer D, MacCallum M A H, Hoenselaers C and Herlt E 2003 Exact Solutions of Einstein's Field Equations (2nd edition, Cambridge: Cambridge University Press)

[6] Bonnor W B 1994 Class. Quantum Grav. 11, 2007

[7] Damour T 1995 Class. Quantum Grav. 12, 725

[8] Bonnor W B 1996 Class. Quantum Grav. 13, 277

[9] Cornish F H J and Micklewright B 1996 Class. Quantum Grav. 13, 2499

[10] Gürses M and Gürsey F 1975 J. Math. Phys. 16, 2385

[11] Cornish F H J and Micklewright B 1996 Class. Quantum Grav. 13, 2505

[12] Dain S, Moreschi O M and Gleiser R J 1996 Class. Quantum Grav. 13, 1155

[13] von der Gönna U and Kramer D 1998 Class. Quantum Grav. 15, 215

[14] Bondi H, van der Burg M G J and Metzner A W K 1962 Proc. Roy. Soc. A 269, 21

[15] Sachs R K 1962 Proc. Roy. Soc. A 270, 103

[16] Cornish F H J and Micklewright B 1999 Gen. Relat. Grav. 31, 913

[17] Cornish F H J and Micklewright B 1999 Class. Quantum Grav. 16, 611

[18] Cornish F H J 2000 Class. Quantum Grav. 17, 3945

[19] Kramer D 1972 Tezisy 3rd Soviet Grav. Conf. (Erevan) p.321

[20] Vaidya P C and Patel L K 1973 Phys. Rev. D 7, 3590

[21] Natorf W and Tafel J 2004 Class. Quantum Grav. 21, 5397

[22] Tafel J and Pukas S 2000 Class. Quantum Grav. 17, 1559

[23] Tafel J 2000 Class. Quantum Grav. 17, 4397

[24] Herlt E 1980 Gen. Relat. Grav. 12, 1

[25] Stephani H 1983 Gen. Relat. Grav. 15, 173

[26] Ivanov B V 1999 J. Math. Phys. 40, 5849

[27] Ivanov B V 1999 Phys. Rev. D 60, 104005

[28] Ivanov B V 2000 Phys. Rev. D 61, 064011

[29] Ivanov B V $1999 g r-q c / 9910072$

[30] Robinson I and Robinson J R 1969 Int. J. Theor. Phys. 2, 231

[31] Trim D W and Wainwright J 1974 J. Math. Phys. 15, 535

[32] Kerr R P and Debney G 1970 J. Math. Phys. 11, 2807

[33] Demiański M 1972 Phys. Lett. A 42, 157 